\title{
Mediastinitis necrotizante descendente de origen dental: revisión de la literatura y presentación de un caso.
}

\author{
Descending necrotizing mediastinitis of dental origin: \\ a case study and review of the literature.
}

\author{
Carlos Alberto Carrasco Rueda,* Ilan Vinitzky Brener, ${ }^{\ddagger}$ Francina Valezka Bolaños Morales, $§$ \\ Zamanta Danaé Zúñiga Garduño, Gerardo Martínez Namorado, "José Antonio Vélez Peralta"
}

RESUMEN

La mediastinitis se define como una inflamación aguda severa de los tejidos conectivos ubicados en la cavidad torácica media, en la que $20 \%$ de los casos puede implicar una infección difusa y polimicrobiana del mediastino denominada mediastinitis necrotizante descendente (MND), secundaria a la propagación de una infección grave desde los tejidos bucofaríngeos o cervicales tales como infecciones odontogénicas (de 36 a $47 \%$ ), faríngeas (de 33 a $45 \%$ ), cervicales (15\%) y otras infecciones de cabeza y cuello (5\%). Clínicamente, los pacientes presentan aumento de volumen, temblores, trismus, odinofagia con disnea, disfagia, hipotensión, dolor de cuerpo y del ángulo de la mandíbula. Puede observarse signo de Hamman (crepitación mediastinal con el latido cardiaco) y enfisema subcutáneo. El manejo quirúrgico de las infecciones odontogénicas, sin importar su severidad, consta de dos principios: eliminar el foco etiológico y el vaciamiento quirúrgico de los espacios anatómicos comprometidos con la instalación de un drenaje adecuado. Se presenta el caso de un masculino de 60 años con diagnóstico de mediastinitis necrotizante descendente de origen dental.

Palabras clave: Mediastinitis descendente, infecciones odontogénicas, extracción dental, complicaciones.

\section{ABSTRACT}

Mediastinitis is defined as a severe acute inflammation of the connective tissues affected in the middle thoracic cavity, in which $20 \%$ of cases may involve a diffuse and polymicrobial infection of the mediastinum, descending necrotizing mediastinitis (MND), secondary to the spread of a serious infection from the oropharyngeal or cervical tissues, stories such as odontogenic infections (36 to 47\%), pharyngeal (33 to 45\%), cervical (15\%) and other head and neck infections (5\%). Clinically, patients present with increased volume, tremors, trismus, odynophagia with dyspnea, dysphagia, hypotension, pain in the body and in the angle of the jaw. Hamman sign (mediastinal crepitus with heartbeat) and subcutaneous emphysema may be observed. The surgical management of odontogenic infections, regardless of their severity, consists of two principles: eliminate the etiological focus and the surgical emptying of the anatomical spaces compromised with the installation of adequate drainage. We present the case of a 60-year-old man diagnosed with descending necrotizing.

Keywords: Descending mediastinitis, odontogenic infections, tooth extraction, complications.

\footnotetext{
* Cirujano dentista Especialista en Cirugía Oral y Maxilofacial. Jefe de Servicio de Estomatología.

¥ Cirujano dentista Especialista en Cirugía Oral y Maxilofacial. Adscrito al Servicio de Estomatología.

§ Médico cirujano Especialista en Cirugía de tórax. Subdirectora del Servicio de Cirugía de Tórax.

I Pasante de Servicio Social en Estomatología.

Instituto Nacional de Enfermedades Respiratorias.
}

Recibido: 15 de septiembre de 2020. Aceptado para publicación: 11 de noviembre de 2020.

Citar como: Carrasco RCA, Vinitzky BI, Bolaños MFV, Zúñiga GZD, Martínez NG, Vélez PJA. Mediastinitis necrotizante descendente de origen dental: revisión de la literatura y presentación de un caso. Rev ADM 2020; 77 (6): 329-336. https://dx.doi.org/10.35366/97623 


\section{INTRODUCCIÓN}

$\mathrm{L}$ a cavidad torácica contiene dos cavidades pleuropulmonares (derecha e izquierda) y un espacio de línea media único llamado mediastino (del latín mediastinus, espacio medio) y puede definirse como el espacio extrapleural comprendido entre ambos pulmones que limita lateralmente con la pleura parietal medial o mediastínica, con el esternón por delante, las vértebras dorsales y los arcos posteriores de las costillas por detrás, el diafragma por debajo y el opérculo torácico por arriba, y contiene el corazón y estructuras que yacen posteriores a él, incluyendo la aorta descendente torácica y el esófago. ${ }^{1}$

La mediastinitis se define como una inflamación aguda severa de los tejidos conectivos ubicados en la cavidad torácica media, en la que $20 \%$ de los casos se derivarán de una infección difusa y polimicrobiana del mediastino denominada mediastinitis necrotizante descendente $(\mathrm{MND}){ }^{2}$ secundaria a la propagación de una infección grave desde los tejidos bucofaríngeos o cervicales ${ }^{3}$ tales como infecciones odontogénicas (de 36 a 47\%), faríngeas (de 33 a 45\%), cervicales (15\%) y otras infecciones de cabeza y cuello (5\%). ${ }^{2}$

La diseminación de la MND puede deberse a propiedades mecánicas, químicas, al gas producido por la bacteria, o al grado de difusión de la bacteria a los tejidos. ${ }^{4}$ El conocimiento de la anatomía de los planos fasciales y de los espacios cervicales ayudará a entender la progresión de estos procesos infecciosos. Existen tres vías potenciales de entrada hacia el mediastino: ${ }^{5}$ (I) la ruta pretraqueal hacia el mediastino anterior; (II) la ruta faríngea lateral al mediastino medio; y (III) la ruta retrofaríngea al mediastino posterior (Figura 1). ${ }^{6}$ Es por ello que la MND se puede clasificar en tres grupos de acuerdo a la extensión anatómica: tipo I = localizada en el mediastino anterior superior, por arriba de la bifurcación traqueal o carina (forma localizada); tipo $I I=$ localizada por debajo de la bifurcación traqueal (forma difusa), la cual es subdivida en: tipo IIA (mediastino anterior inferior) y tipo IIB (mediastino posterior inferior). $2,7-9$

Numerosos reportes indican que pacientes inmunodeprimidos ${ }^{10}$ son más susceptibles a experimentar infecciones odontogénicas graves así como en pacientes de la tercera edad o con enfermedades sistémicas asociadas como diabetes, insuficiencia hepática, insuficiencia renal ${ }^{7}$ y $\mathrm{VIH}$, entre otras. Aunque estas condiciones no son determinantes para el desarrollo del padecimiento, ya que se puede presentar en pacientes sanos. ${ }^{11}$

La MND fue descrita por primera vez en 1938 por Pierse como una enfermedad potencialmente mortal ${ }^{12}$ cuya la tasa de mortalidad reportada en la literatura internacional se mantiene aproximadamente en $40 \%,{ }^{13}$ y cuyo diagnóstico tardío aumenta la mortalidad y la necesidad de una cirugía y reconstrucción más extensas. ${ }^{12}$ La mediastinitis de origen dental fue descrita por Cogan en $1973 .{ }^{14}$ Robles y colaboradores reportan un estudio con 88 casos registrados en el Hospital Juárez de
A

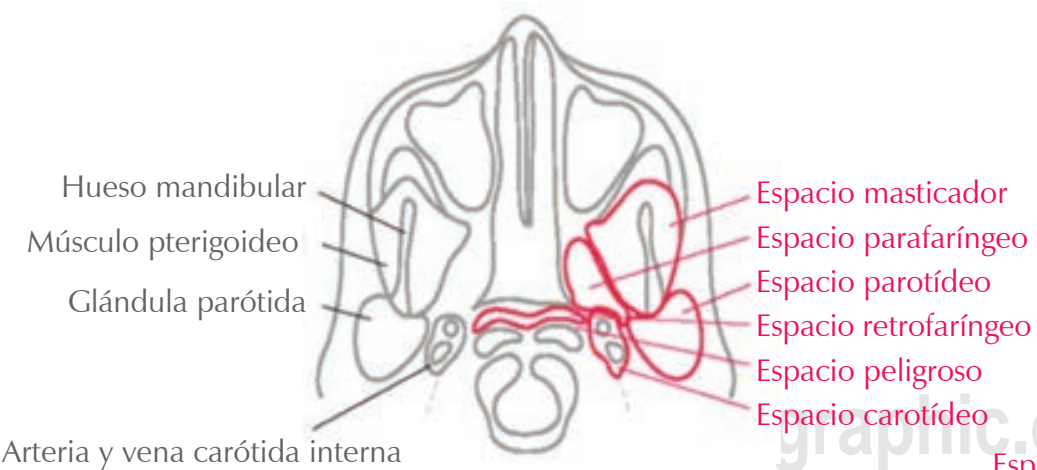

B

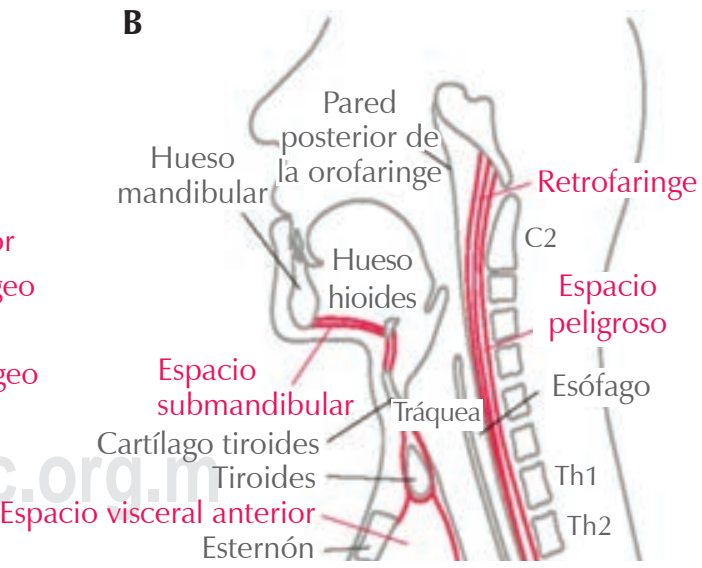

Figura 1: Espacios cervicales importantes para la progresión mediastínica (reproducido de Sumi 2013), «Tratamiento actual para lesiones por quemaduras». A) Vista esquemática axial: el espacio parafaríngeo (sp.) es el centro de las infecciones del espacio profundo que se comunica con los espacios principales: espacio submandibular, espacio retrofaríngeo y espacio carotídeo. B) Vista esquemática sagital: espacio submandibular al mediastino anterior (espacio visceral anterior), espacio retrofaríngeo al mediastino posterior a través del espacio peligroso. ${ }^{6}$ 
México de 2008 a 2017 con una frecuencia de aproximadamente ocho pacientes por año, con predominio en el sexo masculino y un promedio de edad de 51 años. El porcentaje de letalidad fue de $17.6 \%$, el cual disminuyó significativamente a partir de 2013 a 3.1\%, ${ }^{15}$ siendo menor que el reportado en la literatura internacional e igual que el registrado por Prado y su equipo de $18 \%{ }^{2}$ Aunque estos datos contrastan con reportes realizados en años pasados, que describen una letalidad entre 29 y $86 \%$ en los años 80 , aun con tratamiento agresivo. ${ }^{16}$ Lo anterior demuestra la mejora en el conocimiento de la entidad, además de especialistas más y mejor capacitados para el manejo de la patología. En 2014 González y colaboradores publicaron una serie de 2,288 pacientes en el Servicio de Cirugía Cardiovascular del IMSS, los cuales reportaron una incidencia de $2.9 \%$ anual, con una predilección de $71 \%$ en hombres y una mortalidad de $53 \%$, que es superior a la observada en diferentes series publicadas. ${ }^{17}$

Los principales síntomas de la MND son dolor intenso retroesternal (se intensifica durante la respiración y tos), taquipnea, taquicardia, fiebre, infiltración inflamatoria del cuello y tórax. ${ }^{7}$ Clínicamente, los pacientes presentan aumento de volumen local, temblores, trismus, odinofagia con disnea, disfagia, hipotensión, dolor de cuerpo y generalmente en la mandíbula puede observarse el signo de Hamman (crepitación mediastinal con el latido cardiaco) y enfisema subcutáneo. ${ }^{3}$ Es imprescindible conocer el cuadro clínico para identificar de forma temprana la entidad y disminuir la morbimortalidad del paciente. ${ }^{3,18}$ La tomografía computarizada (TC) cervicotorácica es una herramienta invaluable e indispensable en el diagnóstico de MND, tanto en la detección inicial de la infección como en el monitoreo de la respuesta al tratamiento. ${ }^{8}$ La visualización del mediastino ensanchado con múltiples niveles de fluidos y burbujas de aire ectópicas confirman el diagnóstico. ${ }^{3}$ Los exámenes de laboratorio muestran eucocitosis y elevación de la proteína C reactiva. $^{7}$ Se recomienda evaluar el nivel de lactato y la oxigenación mediante gasometría arterial. ${ }^{3,12}$ Los niveles de lactato en la circulación pueden usarse como marcador de hipoperfusión tisular sistémica en pacientes con choque circulatorio y se incluyen ahora en los criterios clínicos de choque séptico definidos en el III Documento Internacional de Consenso para la definición de sepsis y choque séptico (Sepsis-3), los resultados complementan los de otras pruebas como la PCT (procalcitonina) como biomarcador ${ }^{19}$ altamente sensible a inflamación e infección graves..$^{20,21}$
El tratamiento consiste en terapia antibiótica de amplio espectro para cubrir cocos Gram-positivos, bastones Gram-negativos y bacterias anaerobias, ${ }^{22}$ además de soporte vital avanzado, manejo de las vías aéreas, eliminación de los focos sépticos causantes de la patología ${ }^{4}$ y drenaje quirúrgico temprano de las colecciones cervicales y mediastinales. ${ }^{2,4}$ El abordaje de drenaje mediastínico varía ampliamente, a saber, toracotomía, esternotomía media, incisión en concha, abordaje subxifoideo, abordaje transcervical, VATS, mediastinoscopia y drenaje de catéter percutáneo. ${ }^{9}$ Asimismo, soporte alimenticio, monitoreo sistemático de las áreas faciales y prevención de desarrollar focos de infección.

\section{PRESENTACIÓN DEL CASO}

Se presenta el caso de un paciente de 60 años, sin antecedentes médicos relevantes a su ingreso, el cual refiere iniciar padecimiento cuatro días previos al ingreso, presentando odontalgia en región mandibular inferior izquierda, acude con facultativo privado diagnosticando proceso infeccioso de origen dental iniciando tratamiento con ceftriaxona de $1 \mathrm{~g}$ cada 24 horas por tres días de forma parenteral, sin mejoría al tratamiento médico farmacológico, refiriendo exacerbación de sintomatología, por lo que acude al Hospital General del Estado de México, y es referido al Instituto Nacional de Enfermedades Respiratorias, donde ingresa al servicio de urgencias por presentar absceso profundo del cuello complicado con probable mediastinitis. En la valoración inicial muestra un Glasgow de 15, temperatura de 38.5 ${ }^{\circ} \mathrm{C}$, saturación de $\mathrm{O}_{2}$ de $90 \%$ sin oxígeno suplementario, sin datos de dificultad respiratoria, campos pulmonares con ruidos respiratorios normales, abdomen blando, depresible, no doloroso, con mal estado general, fetidez y aumento de volumen y dolor a la palpación en región parotídea y submandibular izquierda así como con hipertermia local, cavidad oral con limitación a la apertura con distancia interincisiva de $20 \mathrm{~mm}$, piso de boca abombado y eritematoso en forma bilateral, caries de tercer grado en segundo molar superior izquierdo y segundo molar inferior izquierdo, halitosis, paladar blando izquierdo abombado y eritematoso.

En tomografía de cuello y tórax en fase simple y contrastada se aprecia densidad similar a gas y enfisema subcutáneo de la región mandibular izquierda (Figura 2), involucrando espacios profundos del cuello (parotídeo izquierdo, masticador izquierdo, parafaríngeo bilateral, retrofaríngeo bilateral, submandibular, submental 


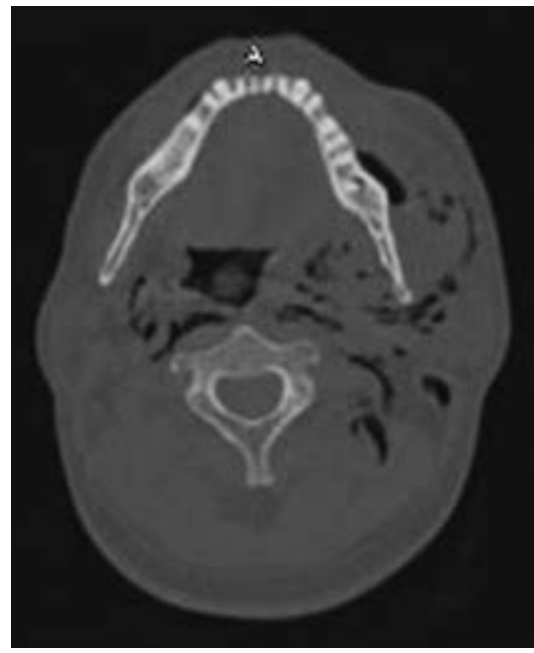

Figura 2: Tomografía axial computarizada corte axial donde se observa aire en el espacio submandibular izquierdo con absceso dental en la raíz del segundo molar inferior izquierdo mandibular (agente causante de la patología).

y sublingual en forma bilateral así como el espacio visceral desde base de cráneo hasta diafragma) (Figura 3); presencia de gas desde del cuello hasta la región mediastinal, presencia de gas en el saco pericárdico, y derrame pleural derecho.

El paciente es ingresado por el servicio de cirugía de tórax, quienes hacen incisión de cervicotomía con salida de material purulento con involucro de la vaina carotídea extendiéndose hasta la base del cráneo y mediastino superior, por la necesidad de realizar esternotomía media, debridamiento de tejido mediastinal pericardiectomía por presencia de pus y gas en el saco pericárdico, además de colocación de sonda pleural derecha.

Se decide colocar sistema VAC (sistema de aspiración cerrada) en región cervical, se realiza valoración por servicio de cirugía maxilofacial del INER, observando un paciente en estado crítico, con una escala de Glasgow de 4 por sedación secundaria a ventilación mecánica asistida, presencia de fetidez debido a proceso infeccioso. A la exploración local se observa presencia de tubo orotraqueal, aumento de volumen e induración en región parotídea, submandibular izquierda y hemicuello izquierdo con hipertermia al tacto. Intraoralmente se aprecia dentición permanente incompleta con múltiples lesiones cariosas de diferentes grados de profundidad, periodontitis crónica generalizada, cálculo dental, halitosis, piso de boca con edema, paladar blando del lado izquierdo con edema y eritema, se observa secreción purulenta al ejercer presión sobre la zona correspondiente a segundos molares superior izquierdo y segundo molar inferior izquierdo. Tórax en tonel con presencia de sonda mediastinal, además de catéter central.

Se intervino quirúrgicamente bajo anestesia general en conjunto con el servicio de cirugía de tórax realizando lavado y debridación $\left(550 \mathrm{~cm}^{3}\right.$ de derrame pleural izquierdo seroso y $100 \mathrm{~cm}^{3}$ de material purulento), cambio de sistema VAC, colocación de sonda endopleural izquierda (Figura 4), odontectomía de segundos molares izquierdos por ser los probables agentes causantes de la patología (Figura 5). Durante el procedimiento se registra salida de $15 \mathrm{~cm}^{3}$ de exudado purulento acumulado en alveolos dentales, se hacen lavados con clorhexidina al $0.12 \%$ + solución fisiológica. Se realiza incisión en fondo de vestíbulo inferior lado izquierdo a la altura de segundo molar, disección roma obteniendo $8 \mathrm{~cm}^{3}$ de material purulento, colocando drenaje Penrose se verifica hemostasia y se continúa con esquema de antibióticos ya establecido por servicio tratante a base de clindamicina y ceftriaxona.

El laboratorio de microbiología reporta desarrollo y crecimiento bacteriano en la muestra (biopsia de cuello) de los siguientes microorganismos (Tabla 1):

\section{Clostridioides difficile \\ Pseudomonas aeruginosa \\ Serratia marcescens}

Se modifica esquema antimicrobiano por meropenem + metronidazol y posteriormente se agrega linezolid y tigeciclina. El paciente se mantiene en $\mathrm{UCl}$ con control hemodinámico estricto y se realizan curaciones diarias

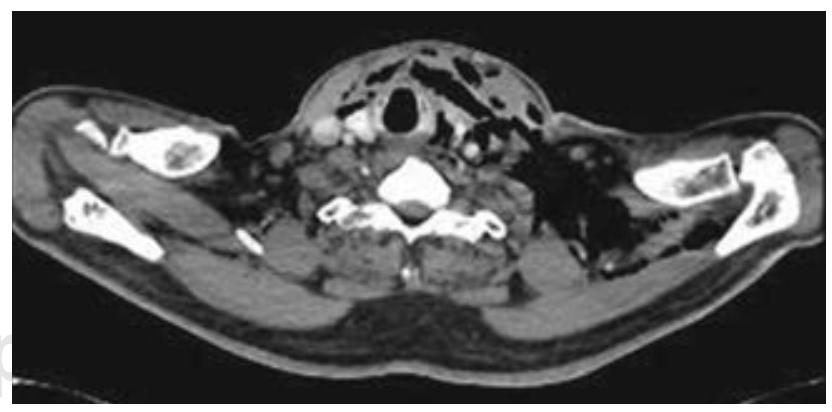

Figura 3: Tomografía axial computarizada corte axial. Zonas hipodensas sugerentes de gas o pus en los espacios profundos del cuello (parotídeo izquierdo, masticador izquierdo, parafaríngeo bilateral, retrofaríngeo bilateral, submandibular, submental y sublingual) en forma bilateral, con desplazamiento de vía aérea. 


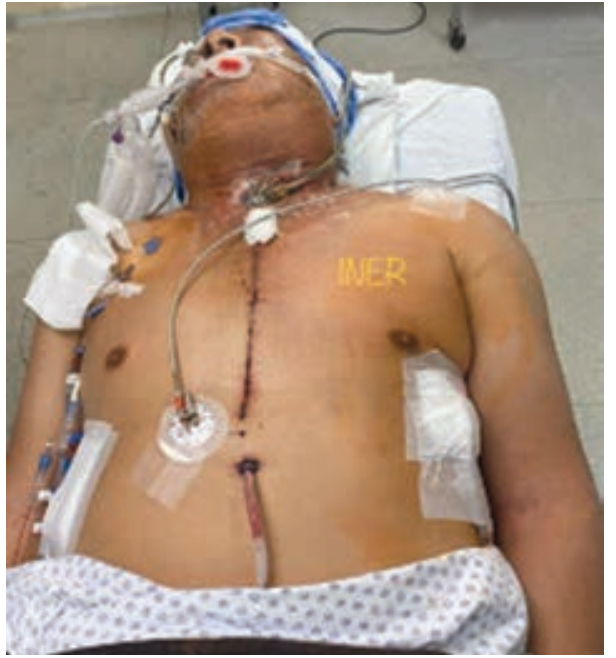

Figura 4: Paciente posterior al tratamiento quirúrgico inicial, observando fasciotomías en cuello con sonda de drenaje, herida por toractomía, sonda pleural e intubación orotraqueal.

intraorales con clorhexidina hasta observar adecuada curación de heridas sin salida de material hematopurulento. A 45 días de su ingreso el paciente se encuentra hemodinámicamente estable, sin datos de proceso infeccioso, extubado y con adecuada ventilación, por lo que se decide su egreso hospitalario para continuar su control por consulta externa. El paciente acude a sus controles en consulta externa de cirugía maxilofacial observando adecuada cicatrización en las zonas intervenidas, aún con higiene oral deficiente, lesiones cariosas de diversos grados y enfermedad periodontal generalizada, por lo que se sugiere iniciar tratamiento estomatológico integral.

\section{DISCUSIÓN}

Las infecciones odontogénicas tienden a extenderse hacia los tejidos que rodean al diente a través de las zonas de menor resistencia, ${ }^{23}$ la MND es potencialmente mortal. ${ }^{13}$ Robles y colaboradore ${ }^{15}$ y Prado y su equipo ${ }^{2}$ señalan un descenso en la tasa de mortalidad de 17.6 a $3.1 \%$ en los últimos siete años así como una recuperación completamente cuando se realiza el tratamiento quirúrgico oportuno, antimicrobianos de espectro adecuado, eliminación de foco infeccioso dental y manejo en unidad de cuidados intensivos. ${ }^{24,25}$ El estado del paciente al ingreso es un factor relevante en la evolución de la enfermedad y los factores asociados a un incremento en la letalidad son: edad avanzada, leucocitosis, enfermedad sistémica asociada como diabetes mellitus, involucramiento del espacio visceral anterior e involucramiento de múltiples espacios, esto comparado con otros pacientes sin los mismos factores de riesgo o asociados. ${ }^{27,28}$

En el presente caso se plantea que el segundo molar inferior izquierdo es el agente causal del proceso infeccioso, el cual es un foco dental bien conocido para infecciones cervicofaciales. La porción apical de los segundos y terceros molares mandibulares alcanzan el punto de origen del músculo milohioideo, por lo que las infecciones periapicales de dichos órganos dentarios pueden extenderse al espacio parafaríngeo o submandibular y así llegar a los espacios profundos del cuello que, junto con la presión intratorácica negativa y la acción de la gravedad, facilita el descenso de la infección hacia la región mediastínica, puesto que ésta no posee ningún techo cerrado que la mantenga aislada del cuello. ${ }^{29}$

Es fundamental en pacientes con sintomatología sugestiva de MND realizar tempranamente una tomografía computarizada, donde se podrá visualizar la colección líquida, a veces con aire ectópico indicativo de la flora bacteriana anaerobia, lo cual permite un abordaje operatorio rápido y dirigido con participación de equipos multidisciplinarios. Posterior al tratamiento quirúrgico inicial se sugiere realizar una TC de control para verificar la evolución y la necesidad o no de un segundo drenaje. ${ }^{30,31}$

En el presente caso las pruebas de cultivo revelaron una microbiota mixta compuesta por: Pseudomonas aeruginosa, Serratia marcescens, Clostridioides difficile, lo

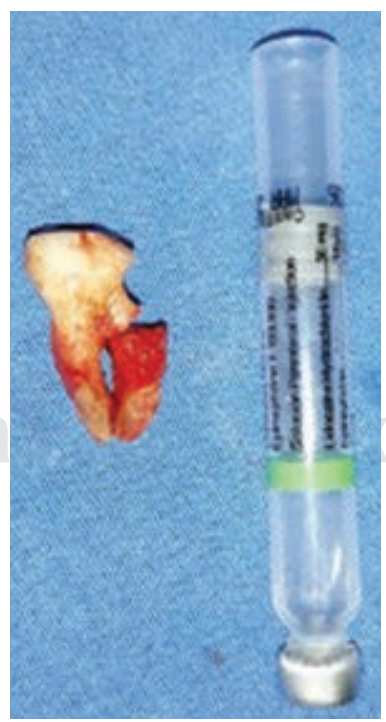

Figura 5:

Segundo molar inferior izquierdo extraído con datos de caries profunda, etiología de la mediastinitis. 
Tabla 1: Microorganismos presentes en mediastinitis necrotizante descendente.

\begin{tabular}{|c|c|c|}
\hline & Bacterias aeróbicas & Bacterias anaerobias \\
\hline Carrasco et al. & Pseudomonas aeruginosa & $\begin{array}{l}\text { Serratia marcescens } \\
\text { Clostridioides difficile }\end{array}$ \\
\hline González R, Risco R ${ }^{3}$ & Streptococcus viridans & Prevotella spp. \\
\hline Sumi $Y^{6}$ & $\begin{array}{l}\text { Streptococcus constellatus, Streptococcus interme- } \\
\text { dius, Streptococcus agalactiae, Streptococcus mitis, } \\
\text { Corynebacterium, Klebsiella pneumonia }\end{array}$ & $\begin{array}{l}\text { Staphylococcus aureus } \\
\text { Peptostreptococcus, Bacteroides fragilis, } \\
\text { Prevotella y Fusobacterium }\end{array}$ \\
\hline Fukuchi M., Suzuki O, Nasu D ${ }^{8}$ & Streptococcus constellatus & Staphylococcus aureus, Streptococcus pyogenes \\
\hline De Oliveira E, Teixeira $\mathrm{R}^{13}$ & Pseudomonas spp. & $\begin{array}{l}\text { Staphylococcus aureus, Staphylococcus } \\
\text { epidermidis, Escherichia coli, Eubacterium, } \\
\text { Lactobaciullus spp. }\end{array}$ \\
\hline Filiaci $\mathrm{F}^{22}$ & Streptococcus constellatus & $\begin{array}{l}\text { Streptococcus sanguis, Streptococcus mutans, } \\
\text { Actinomyces viscosus, Streptococcus pneumo- } \\
\text { niae, Veillonella párvula }\end{array}$ \\
\hline Brook I, Frazier $\mathrm{EH}^{33}$ & $\begin{array}{l}\text { Streptococcus constellatus, Streptococcus interme- } \\
\text { dius, Streptococcus agalactiae, Streptococcus mitis }\end{array}$ & $\begin{array}{l}\text { Peptostreptococcus, Bacteroides fragilis, } \\
\text { Prevotella y Fusobacterium }\end{array}$ \\
\hline Lewandowski B, Paklan $\mathrm{P}^{34}$ & $\begin{array}{l}\text { Acinetobacter baumannii, Streptococcus anginosus, } \\
\text { Candida albicans }\end{array}$ & $\begin{array}{l}\text { Klebsiella pneumoniae, Enterococcus fae- } \\
\text { cium, Veillonella ssp. }\end{array}$ \\
\hline Deu-Martín M, Saez M² & Pseudomonas aeruginosa, Streptococcus viridans & $\begin{array}{l}\text { Bacteroides spp, Clostridioides difficile, Esch- } \\
\text { erichia coli, Fusobacterium avium, Strepto- } \\
\text { coccus pyogenes }\end{array}$ \\
\hline Rodriguez S, Fortún H, Lleonart P ${ }^{34}$ & $\begin{array}{l}\text { Peptoestreptococo fusobacterium, Haemophilus } \\
\text { influenzae, Candida albicans }\end{array}$ & $\begin{array}{l}\text { Streptococo beta hemolítico grupo A, Strep- } \\
\text { tococos viridans, Bacteroide melaninógeno, } \\
\text { Staphylococcus aureus }\end{array}$ \\
\hline
\end{tabular}

cual a pesar de no representar la microbiota bacteriana común de estas infecciones coincide con lo reportado por Deu-Martín M. y Saez-Barba $\mathrm{M}^{32}$ sobre los agentes causales de la MND.

Tabla 2: Principios para el tratamiento de infecciones odontogénicas. ${ }^{26}$

I. Determinar la etiología y severidad de la infección

II. Evaluar los antecedentes médicos y sistema inmunológico del paciente

III. Determinar si la atención será en hospital o ambulatoria

IV. Tratamiento quirúrgico

V. Soporte médico y nutricional

VI. Tratamiento antimicrobiano empírico (espectro adecuado) y posteriormente específico

VII. Evaluar al paciente periódicamente
Uno de los principios de la terapéutica antibiótica se basa en el conocimiento de los agentes causantes de la infección. El reconocimiento de la flora mixta así como la buena respuesta general a la antibioterapia administrada son elementos que justifican el tratamiento antibiótico empírico de estos procesos. Sin embargo, hay que tener en cuenta la necesidad de recoger muestras para cultivo y antibiograma ante determinadas situaciones como inmunodepresión, sospecha de osteomielitis o infecciones recidivantes $^{33}$ (Tabla 2). En el presente caso se inició con un manejo antimicrobiano empírico, el cual se fue modificando con base en los resultados de cultivo y la respuesta del paciente.

\section{CONCLUSIONES}

La MND es una complicación poco frecuente, pero potencialmente mortal de una infección dental. Los médicos y odontólogos debemos ser capaces de 
reconocer los factores predisponentes, los signos y síntomas para poder referir oportunamente al paciente a un centro especializado que cuente con especialistas en cirugía maxilofacial y cirugía de tórax así como unidad de cuidados intensivos. El diagnóstico precoz al igual que un espectro adecuado y la eliminación del foco dental causal son la clave para un mejor pronóstico para los pacientes con MND. La prevención debe ser el pilar fundamental en la práctica médica y odontológica actual, lo cual evita que los pacientes puedan llegar a estados críticos como el descrito en el presente artículo.

\section{BIBLIOGRAFÍA}

1. Hansen J. Netter's clinical anatomy. Journal of chemical information and modeling. 4th ed. Vol. 53, New York: Elsevier; 2018, pp. 16891699.

2. Prado H, Jiménez E, Jiménez I. Descending necrotizing mediastinitis: systematic review on its treatment in the last 6 years, 75 years after its description. Head Neck. 2016; 36 (10): 2275-2283.

3. González-García R, Risco-Rojas R, Román-Romero L, MorenoGarcía C, López García C. Descending necrotizing mediastinitis following dental extraction. Radiological features and surgical treatment considerations. J Cranio-Maxillofacial Surg. 2011; 39 (5): 335-339. Available from: http://dx.doi.org/10.1016/j. jcms.2010.10.001.

4. Petitpas F, Blancal JP, Mateo J, Farhat I, Naija W, Porcher R et al. Factors associated with the mediastinal spread of cervical necrotizing fasciitis. Ann Thorac Surg. 2012; 93 (1): 234-248. Available from: http://dx.doi.org/10.1016/j.athoracsur.2011.09.012.

5. Pinto A, Scaglione M, Scuderi MG, Tortora G, Daniele S, Romano L. Infections of the neck leading to descending necrotizing mediastinitis: role of multidetector row computed tomography. Eur J Radiol. 2008; 65 (3): 389-394.

6. Sumi Y. Descending necrotizing mediastinitis: 5 years of published data in Japan. Acute Med Surg. 2015; 2 (1): 1-12.

7. Watanabe H, Osano H, lida H, Hayashi H, Dohi A, Mori Y. A case of descending necrotizing mediastinitis secondary to odontogenic infection. J Oral Maxillofac Surgery, Med Pathol. 2019; 31 (2): 126130. Available from: https://doi.org/10.1016/j.ajoms.2018.11.002.

8. Fukuchi M, Suzuki O, Nasu D, Koizumi K, Muta Y, Imaizumi H et al. Descending necrotizing mediastinitis treated with tooth extractions following mediastinal and cervical drainage. Case Rep Gastroenterol. 2015; 9 (3): 311-316.

9. Endo S, Murayama F, Hasegawa T, Yamamoto S, Yamaguchi T, Sohara $Y$ et al. Guideline of surgical management based on diffusion of descending necrotizing mediastinitis. Jpn J Thorac Cardiovasc Surg. 1999; 47: 14-19.

10. Diamantis S, Giannakopoulos H, Chou J, Foote J. Descending necrotizing mediastinitis as a complication of odontogenic infection. Int J Surg Case Rep. 2011; 2 (5): 65-67. Available from: http:// dx.doi.org/10.1016/j.ijscr.2011.01.004.

11. Jansisyanont P, Kasemsai W, Bamroong P. Factors related to the treatment outcome of maxillofacial fascia space infection. J Oral Maxillofac Surgery, Med Pathol. 2015; 27 (4): 458-464. Available from: http://dx.doi.org/10.1016/j.ajoms.2014.04.009.
12. Panda NK, Simhadri S, Rao Sridhara S. Cervicofacial necrotizing fasciitis: Can we expect a favourable outcome? J Laryngol Otol. 2004; 118 (10): 771-777.

13. Martins E, Junior DO, Teixeira R, Cerqueira T. Descending necrotizing mediastinitis secondary to a dental infection. Autops Case Rep. 2012; 2 (1): 37-42.

14. Cogan IM. Necrotizing mediastinitis secondary to descending cervical cellulitis. Oral Surg Oral Med Oral Pathol. 1973; 36 (3): 307-320.

15. Robles-Pérez E, Córdova-López JL, López-Rodríguez R, RamosCano VH, Robles-Pérez VE, Zamora-Gómez SE et al. Factores que inciden en la mortalidad de la mediastinitis aguda necrosante descendente. Rev Hosp Jua Mex. 2018; 85 (3): 127-135.

16. Estrera AS, Landay MJ, Grishman JM, Sinn DP, Platt MR. Descending necrotizing mediastinitis. Surg Gynecol Obstet. 1983; 157 (6): 542552.

17. González FS, Molina GJ, Villela ML. Factores de riesgo y mortalidad asociada a mediastinitis. Enf Inf Microbiol. 2014; 34 (1): 13-25.

18. Fabio F. Disseminated necrotic mediastinitis spread from odontogenic abscess: our experience. Ann Stomatol (Roma). 2015; (2): 64-68.

19. Bolaños F. Procalcitonina semicuantitativa y score de SOFA y APACHE en el pronóstico de pacientes con mediastinitis. Revista Respirar 5-7 Congreso ALAT 2012.

20. Singer M, Deutschman CS, Seymour C, Shankar-Hari M, Annane $\mathrm{D}$, Bauer $\mathrm{M}$ et al. The Third International Consensus Definitions for Sepsis and Septic Shock (Sepsis-3). JAMA. 2016; 315 (8): 801-810.

21. Becker KL, Snider R, Nylen ES. Procalcitonin assay in systemic inflammation, infection, and sepsis: clinical utility and limitations. Crit Care Med. 2008; 36 (3): 941-952.

22. Cirino L, Elias F, Almeida J. Descending mediastinitis. Sao Paulo Med J. 2006; 124 (5): 285-290.

23. Kang SK, Lee $\mathrm{S}$, Oh HK, Kang MW, Na MH, Yu JH et al. Clinical features of deep neck infections and predisposing factors for mediastinal extension. Korean J Thorac Cardiovasc Surg. 2012; 45: 171-176.

24. Poeschl W, Spusta L, Russmueller G, Seemann R, Hirschl A, Poeschl $E$ et al. Antibiotic susceptibility and resistance of the odontogenic microbiological spectrum and its clinical impact on severe deep space head and neck infections. Oral Surg Oral Med Oral Pathol Oral Radiol Endod. 2010; 110: 151-156.

25. D'Cunha J, James M, Antonoff MB, Green CA, Andrade RS, Maddaus MA et al. Descending necrotizing mediastinitis: a modified algorithmic approach to define a new standard of care. Surg Infect (Larchmt). 2013; 14 (6): 525-531.

26. Peterson LJ. Contemporary management of deep infec- tions of the neck. J Oral Maxillofac Surg. 1993; 51: 226-231.

27. Tung-Yiu W, Jehn-Shyun H, Ching-Hung C, Hung-An C. Cervical necrotizing fasciitis of odontogenic origin: a report of 11 cases. J Oral Maxillofac Surg. 2000; 58: 1347-1352.

28. Boscolo-Rizzo P, Marchiori C, Montolli F, Vaglia A, Da Mosto MC. Deep neck infections: a constant challenge. ORL J Otorhinolaryngol Relat Spec. 2006; 68 (5): 259-265.

29. Edwards JD, Sadeghi N, Najam F, Margolis M. Craniocervical necrotizing fasciitis of odontogenic origin with mediastinal extension. Ear Nose Throat J. 2004; 83: 579-582.

30. Yang X, Soimakallio S. The role of different imaging procedures on early diagnosis and management of descending necrotizing mediastinitis. Eur Arch Otorhinolaryngol. 1996; 253: 316-317.

31. Honguero A, Arnau A, Fernández CA, Saumench PR, Estors AM, Cantó AA. Descending necrotizing mediastinitis: treatment by 
transcervical thoracic drainage. Arch Bronconeumol. 2005; 41 (5): 293-294.

32. Deu-Martín M, Saez-Barba M, López-Sanz I, Alcaraz-Peñarrocha $\mathrm{R}$, Romero-Vielva L, Solé-Montserrat J. Factores de riesgo de mortalidad en la mediastinitis necrosante descendente. Arch Bronconeumol. 2010; 46 (4): 182-187.

33. Brook I, Frazier E. Microbiology of mediastinitis. Arch Intern Med. 1996; 156: 333-336.

34. Lewandowski B, Pakla P, Wołek W, Jednakiewicz M, Nicpoń J. A fatal case of descending necrotizing mediastinitis as a complication of odontogenic infection. A case report. Kardiochir Torakochirurgia Pol. 2014; 11 (3): 324-328.

\section{Correspondencia:}

Dr. Carlos A. Carrasco Rueda

E-mail: drcarrascor@hotmail.com

Conflicto de intereses: Los autores declaran no tener ningún conflicto de intereses. 